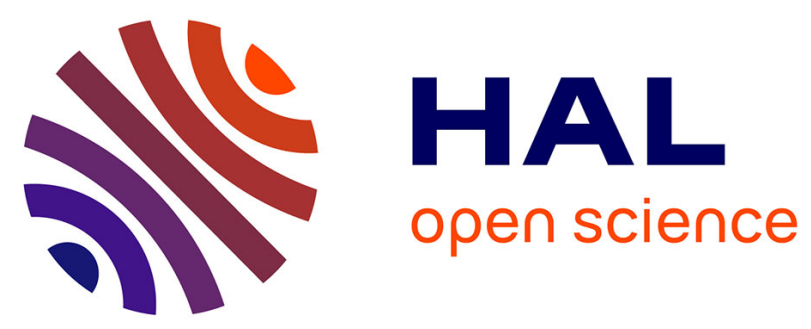

\title{
Large System Analysis of Zero-Forcing Precoding in MISO Broadcast Channels with Limited Feedback
}

\author{
Sebastian Wagner, Romain Couillet, Dirk Slock, Merouane Debbah
}

\section{To cite this version:}

Sebastian Wagner, Romain Couillet, Dirk Slock, Merouane Debbah. Large System Analysis of ZeroForcing Precoding in MISO Broadcast Channels with Limited Feedback. 11th IEEE International Workshop on Signal Processing Advances in Wireless Communications, Jun 2010, Marrakech, Morocco. pp.1 - 5, 10.1109/SPAWC.2010.5671035 . hal-00675528

\section{HAL Id: hal-00675528 \\ https://hal.science/hal-00675528}

Submitted on 1 Mar 2012

HAL is a multi-disciplinary open access archive for the deposit and dissemination of scientific research documents, whether they are published or not. The documents may come from teaching and research institutions in France or abroad, or from public or private research centers.
L'archive ouverte pluridisciplinaire $\mathbf{H A L}$, est destinée au dépôt et à la diffusion de documents scientifiques de niveau recherche, publiés ou non, émanant des établissements d'enseignement et de recherche français ou étrangers, des laboratoires publics ou privés. 


\title{
Large System Analysis of Zero-Forcing Precoding in MISO Broadcast Channels with Limited Feedback
}

\author{
Sebastian Wagner* ${ }^{* \dagger}$, Romain Couillet ${ }^{* \ddagger}$, Dirk T. M. Slock ${ }^{\dagger}$, and Mérouane Debbah ${ }^{\ddagger}$ \\ *ST-ERICSSON, 06904 Sophia-Antipolis, France, Email: \{sebastian.wagner, romain.couillet\}@ stericsson.com \\ †EURECOM, 06904 Sophia-Antipolis, France, Email: \{sebastian.wagner, dirk.slock\}@eurecom.fr \\ ${ }_{\ddagger}^{\ddagger}$ SUPÉLEC, 91192 Gif sur Yvette, France, Email: \{romain.couillet, merouane.debbah\}@ supelec.fr
}

\begin{abstract}
In this paper we analyze the sum rate of zero-forcing (ZF) precoding in MISO broadcast channels with limited feedback, transmit correlation and path loss. Our analysis assumes that the number of transmit antennas $M$ and the number of users $K$ are large, while their ratio remains bounded. By applying recent results from random matrix theory we derive a deterministic equivalent of the signal-to-interference plus noise ratio (SINR) and compute the sum rate maximizing number of users as well as the limiting sum rate for high signal-to-noise ratios (SNR), as a function of the channel errors and the channel correlation pattern. Simulations show that theoretical and numerical results match well, even for small system dimensions.
\end{abstract}

\section{INTRODUCTION}

The capacity achieving precoding strategy of the Gaussian MIMO broadcast channel based on the non-linear dirty-paper coding (DPC) technique [1]. But so far no efficient practical algorithm implementing the optimal DPC scheme has been proposed. Therefore, low complexity linear precoding strategies have gained a lot of attention since they achieve a large portion of the rate region at moderate complexity. A classical linear interference mitigating scheme is zero-forcing (ZF) precoding which has first been analyzed in the context of MIMO broadcast channels in [2].

In this contribution we consider a system where both the number of transmit antennas $M$ and the number of users $K$ are large but their ratio $\beta(M) \triangleq M / K$ is bounded. We extend the models of [3]-[5] by considering imperfect channel state information at the transmitter (CSIT), transmit correlation as well as different path losses of the users. With the aid of recent tools from random matrix theory (RMT), we derive a deterministic equivalent of the signal-to-interference plus noise ratio (SINR) of $\mathrm{ZF}$ precoding which is independent of the individual channel realizations. From the deterministic equivalent of the SINR we determine the sum rate maximizing number of users.

Notation: In the following boldface lower-case and uppercase characters denote vectors and matrices, respectively. The operators $(\cdot)^{\mathrm{H}}, \operatorname{tr}(\cdot)$ and, for $\mathbf{X}$ of size $N \times N, \operatorname{Tr}(\mathbf{X}) \triangleq \frac{1}{N} \operatorname{tr} \mathbf{X}$ denote conjugate transpose, trace and normalized matrix trace, respectively. The expectation is $E[\cdot]$ and $\operatorname{diag}\left(x_{1}, \ldots, x_{N}\right)$ is the diagonal matrix with elements $x_{i}$ on the main diagonal.
The $N \times N$ identity matrix is $\mathbf{I}_{N}$ and $\Im[z]$ is the imaginary part of $z \in \mathbb{C}$.

\section{Mathematical Preliminaries}

Definition 1 (Deterministic Equivalent): Let $\left\{\mathbf{X}_{N}: N=\right.$ $1,2, \ldots\}$ be a set of complex random matrices $\mathbf{X}_{N}$ of size $N \times$ $N$. For some functional $f$ we define a deterministic equivalent $m_{\mathbf{X}_{N}}^{\circ}$ of $m_{\mathbf{X}_{N}} \triangleq f\left(\mathbf{X}_{N}\right)$ as any series $m_{\mathbf{X}_{1}}^{\circ}, m_{\mathbf{X}_{2}}^{\circ}, \ldots$ such that

$$
m_{\mathbf{X}_{N}}-m_{\mathbf{X}_{N}}^{\circ} \stackrel{N \rightarrow \infty}{\longrightarrow} 0
$$

almost surely.

In present work we are interested in deterministic equivalents of expressions of the form

$$
m_{\mathbf{B}_{N}, \mathbf{Q}_{N}}(z)=\operatorname{Tr} \mathbf{Q}_{N}\left(\mathbf{B}_{N}-z \mathbf{I}_{n}\right)^{-1}
$$

where $\mathbf{Q}_{N} \in \mathbb{C}^{n \times n}$ is a Hermitian positive definite matrix and $\mathbf{B}_{N} \in \mathbb{C}^{n \times n}$ is of the type

$$
\mathbf{B}_{N}=\mathbf{T}_{N}^{1 / 2} \mathbf{X}_{N} \mathbf{R}_{N} \mathbf{X}_{N}^{\mathrm{H}} \mathbf{T}_{N}^{1 / 2}
$$

where $\mathbf{R}_{N} \in \mathbb{C}^{N \times N}$ is nonnegative definite Hermitian, $\mathbf{T}_{N} \in \mathbb{C}^{n \times n}$ is diagonal and $\mathbf{X}_{N} \in \mathbb{C}^{n \times N}$ is random with independent and identically distributed (i.i.d.) entries of zero mean and variance $1 / n$. In the course of the derivations, we will require the following result,

Theorem 1: [6, Theorem 1] Under the above model for $\mathbf{B}_{N}$ where $\mathbf{T}_{N}$ and $\mathbf{R}_{N}, \mathbf{Q}_{N}$ have uniformly bounded spectral norm (w.r.t. $N$ ), as $(n, N)$ grow large with ratio $\beta(N) \triangleq N / n$ such that $0<\liminf _{N} \beta(N) \leq \lim \sup _{N} \beta(N)<\infty$. Define $m_{\mathbf{B}_{N}, \mathbf{Q}_{N}}(z)$ as in (2). Then, for $z \in \mathbb{C} \backslash \mathbb{R}^{+}$,

$$
m_{\mathbf{B}_{N}, \mathbf{Q}_{N}}(z)-m_{\mathbf{B}_{N}, \mathbf{Q}_{N}}^{\circ}(z) \stackrel{N \rightarrow \infty}{\longrightarrow} 0
$$

almost surely, where $m_{\mathbf{B}_{N}, \mathbf{Q}_{N}}^{\circ}(z)$ is defined as

$$
\begin{aligned}
m_{\mathbf{B}_{N}, \mathbf{Q}_{N}}^{\circ}(z) & =\operatorname{Tr} \mathbf{Q}_{N}\left(c(z) \mathbf{T}_{N}-z \mathbf{I}_{n}\right)^{-1} \\
\text { with } \quad c(z) & =\operatorname{Tr} \mathbf{R}_{N}\left(\mathbf{I}_{N}+\frac{1}{\beta} e(z) \mathbf{R}_{N}\right)^{-1}
\end{aligned}
$$

and $e(z)$ is the unique solution of

$$
e(z)=\operatorname{Tr} \mathbf{T}_{N}\left(c(z) \mathbf{T}_{N}-z \mathbf{I}_{n}\right)^{-1}
$$


with positive imaginary part if $\Im[z]>0$, of negative imaginary part if $\Im[z]<0$, and real positive if $z<0$. Moreover $e(z)$ is analytic on $\mathbb{C} \backslash \mathbb{R}^{+}$and of uniformly bounded module on every compact subset of $\mathbb{C} \backslash \mathbb{R}^{+}$. Note that $m_{\mathbf{B}_{N}}(z) \triangleq m_{\mathbf{B}_{N}, \mathbf{I}_{N}}(z)$ is the Stieltjes transform [7] of the eigenvalue distribution of $\mathbf{B}_{N}$.

\section{System Model}

Consider the MISO broadcast channel composed of one central transmitter equipped with $M$ antennas and of $K$ single-antenna receivers. Assume $M>K$ and narrow-band communication. Denoting $y_{k}$ the signal received by user $k$, the concatenated received signal vector $\mathbf{y}=\left[y_{1}, \ldots, y_{K}\right]^{\top} \in \mathbb{C}^{K}$ at a given time instant reads

$$
\mathbf{y}=\sqrt{M} \mathbf{H} \mathbf{x}+\mathbf{n}
$$

with transmit vector $\mathbf{x} \in \mathbb{C}^{M}$, channel matrix $\mathbf{H} \in \mathbb{C}^{K \times M}$ and noise vector $\mathbf{n} \sim \mathcal{C N}\left(0, \sigma^{2} \mathbf{I}_{K}\right)$. The transmit vector $\mathbf{x}$ is obtained by linear precoding $\mathbf{x}=\mathbf{G s}$, where $\mathbf{s} \sim \mathcal{C N}\left(0, \mathbf{I}_{K}\right)$ is the symbol vector and $\mathbf{G}=\left[\mathbf{g}_{1}, \ldots, \mathbf{g}_{K}\right] \in \mathbb{C}^{M \times K}$ is the precoding matrix. The total transmit power is $P>0$, hence

$$
\operatorname{tr}\left(E\left[\mathbf{x x}^{\mathrm{H}}\right]\right)=\operatorname{tr}\left(\mathbf{G G}^{\mathrm{H}}\right) \leq P .
$$

In this paper we consider ZF precoding i.e.

$$
\mathbf{G}=\frac{\xi}{\sqrt{M}}\left(\hat{\mathbf{H}}^{\mathrm{H}} \hat{\mathbf{H}}\right)^{-1} \hat{\mathbf{H}}^{\mathrm{H}}
$$

where $\hat{\mathbf{H}}$ is the estimated channel matrix available at the transmitter and the scaling factor $\xi$ is set to fulfill the power constraint (9). From (9) we then obtain

$$
\xi^{2}=\frac{P}{\frac{1}{M} \operatorname{tr}\left(\hat{\mathbf{H}}^{\mathrm{H}} \hat{\mathbf{H}}\right)^{-1}}=\frac{P}{m_{\hat{\mathbf{H}}^{\mathrm{H}} \hat{\mathbf{H}}}(0)} \triangleq \frac{P}{\bar{\Psi}} .
$$

The received symbol $y_{k}$ of user $k$ is given by

$$
y_{k}=\xi \mathbf{h}_{k}^{\mathrm{H}}\left(\hat{\mathbf{H}}^{\mathrm{H}} \hat{\mathbf{H}}\right)^{-1} \hat{\mathbf{h}}_{k} s_{k}+\xi \sum_{i=1, i \neq k}^{K} \mathbf{h}_{k}^{\mathrm{H}}\left(\hat{\mathbf{H}}^{\mathrm{H}} \hat{\mathbf{H}}\right)^{-1} \hat{\mathbf{h}}_{i} s_{i}+n_{k}
$$

where $\mathbf{h}_{k}^{\mathrm{H}}$ and $\hat{\mathbf{h}}_{k}^{\mathrm{H}}$ denote the $k$ th row of $\mathbf{H}$ and $\hat{\mathbf{H}}$, respectively. The SINR $\gamma_{k, \mathrm{zf}}$ of user $k$ can be written in the form

$$
\gamma_{k, \mathrm{zf}}=\frac{\left|\mathbf{h}_{k}^{\mathrm{H}}\left(\hat{\mathbf{H}}^{\mathrm{H}} \hat{\mathbf{H}}\right)^{-1} \hat{\mathbf{h}}_{k}\right|^{2}}{\mathbf{h}_{k}^{\mathrm{H}}\left(\hat{\mathbf{H}}^{\mathrm{H}} \hat{\mathbf{H}}\right)^{-1} \hat{\mathbf{H}}_{[k]}^{\mathrm{H}} \hat{\mathbf{H}}_{[k]}\left(\hat{\mathbf{H}}^{\mathrm{H}} \hat{\mathbf{H}}\right)^{-1} \mathbf{h}_{k}+\frac{\bar{\Psi}}{\rho}}
$$

where $\hat{\mathbf{H}}_{[k]}^{\mathrm{H}}=\left[\hat{\mathbf{h}}_{1}, \ldots, \hat{\mathbf{h}}_{k-1}, \hat{\mathbf{h}}_{k+1}, \ldots, \hat{\mathbf{h}}_{K}\right] \in \mathbb{C}^{M \times(K-1)}$ and $\rho=P / \sigma^{2}$ denotes the signal-to-noise ratio (SNR). The sum rate $R_{\text {sum }}$ is given by

$$
R_{\text {sum }}=\sum_{k=1}^{K} \log \left(1+\gamma_{k, \mathrm{zf}}\right) \quad[\text { nats } / \mathrm{s} / \mathrm{Hz}] .
$$

Under the assumption of a rich scattering environment the correlated channel can be modeled as [8]-[10]

$$
\mathbf{H}=\mathbf{L}^{1 / 2} \mathbf{X} \Theta^{1 / 2}
$$

where $\mathbf{X} \in \mathbb{C}^{K \times M}$ has i.i.d. zero-mean entries of variance $1 / M, \Theta \in \mathbb{C}^{M \times M}$ is the nonnegative definite correlation matrix at the transmitter with eigenvalues $\lambda_{1}, \ldots, \lambda_{M}$, ordered as $\lambda_{1} \leq \ldots \leq \lambda_{M}$, and $\mathbf{L}=\operatorname{diag}\left(l_{1}, \ldots, l_{K}\right)$, with entries ordered as $l_{1} \leq \ldots \leq l_{K}$, contains the user channel gains, i.e. the inverse user path losses. Note that in (14) the entries of $\mathbf{X}$ are not required to be Gaussian. We assume $\|\mathbf{\Theta}\|$ to be uniformly bounded from above with respect to $M$, i.e. adding more transmit antennas does not significantly increase the correlation between them.

Moreover, we suppose that only $\hat{\mathbf{H}}$, an imperfect estimate of the true channel matrix $\mathbf{H}$, is available at the transmitter. The channel gain matrix $\mathbf{L}$ as well as the transmit correlation $\Theta$ are assumed to be slowly varying compared to the channel coherence time and are assumed to be perfectly known to the transmitter. We therefore model $\hat{\mathbf{H}}$ as

$$
\begin{aligned}
\hat{\mathbf{H}} & =\mathbf{L}^{1 / 2} \hat{\mathbf{X}} \boldsymbol{\Theta}^{1 / 2} \\
\text { with } \quad \hat{\mathbf{X}} & =\sqrt{1-\tau^{2}} \mathbf{X}+\tau \mathbf{Q}
\end{aligned}
$$

where $\mathbf{Q} \in \mathbb{C}^{K \times M}$ is the matrix of channel estimation errors containing i.i.d. entries of zero mean and variance $1 / M$, and $\tau \in[0,1]$. The parameter $\tau$ reflects the amount of distortion in the channel estimate $\hat{\mathbf{H}}$. Furthermore, we suppose that $\mathbf{X}$ and $\mathbf{Q}$ are mutually independent as well as independent of the symbol vector $\mathbf{s}$ and noise $\mathbf{n}$. A similar model for imperfect CSIT has been used in [11]-[13].

\section{Deterministic Equivalent of the SINR}

In the following we derive a deterministic equivalent $\gamma_{k, \mathrm{zf}}^{\circ}$ of the SINR $\gamma_{k, \mathrm{zf}}$ of user $k$ for ZF precoding. That is, $\gamma_{k, \mathrm{zf}}^{\circ}$ is an approximation of $\gamma_{k, \mathrm{zf}}$ independent of the particular realizations of $\mathbf{X}$ and $\mathbf{Q}$.

In [6] we derived a deterministic equivalent for regularized ZF (RZF) precoding. The same techniques as for RZF, cannot be applied for ZF, since by removing a row of $\hat{\mathbf{H}}$ the matrix $\hat{\mathbf{H}}^{\mathrm{H}} \hat{\mathbf{H}}$ becomes singular. Therefore, we adopt a different strategy and derive the SINR $\gamma_{k, \mathrm{zf}}$ for ZF of user $k$ and $\beta>1$ as $\gamma_{k, \mathrm{zf}}=\lim _{\alpha \rightarrow 0} \gamma_{k, \mathrm{rzf}}$. The result is summarized in the following theorem.

Theorem 2: Let $\beta>1$ and $\gamma_{k, \mathrm{zf}}$ be the SINR of user $k$ for $\mathrm{ZF}$ precoding. Then

$$
\gamma_{k, \mathrm{zf}}-\gamma_{k, \mathrm{zf}}^{\circ} \stackrel{M \rightarrow \infty}{\longrightarrow} 0
$$

almost surely, where $\gamma_{k, \mathrm{zf}}^{\circ}$ is a deterministic equivalent of $\gamma_{k, \mathrm{zf}}$ and given by

$$
\gamma_{k, \mathrm{zf}}^{\circ}=\frac{1-\tau^{2}}{l_{k} \tau^{2} \bar{\Upsilon}^{\circ}+\frac{\bar{\Psi}^{\circ}}{\rho}}
$$

with

$$
\begin{aligned}
\bar{\Psi}^{\circ} & =\frac{1}{\beta \bar{c}} \operatorname{Tr} \mathbf{L}^{-1} \\
\bar{\Upsilon}^{\circ} & =\frac{c_{2} / \bar{c}^{2}}{\beta-c_{2} / \bar{c}^{2}} \operatorname{Tr} \mathbf{L}^{-1} \\
c_{2} & =\operatorname{Tr} \boldsymbol{\Theta}^{2}\left(\mathbf{I}_{M}+\frac{1}{\bar{c} \beta} \boldsymbol{\Theta}\right)^{-2}
\end{aligned}
$$


where $\bar{c}$ is the unique solution of

$$
\bar{c}=\operatorname{Tr} \Theta\left(\mathbf{I}_{M}+\frac{1}{\bar{c} \beta} \boldsymbol{\Theta}\right)^{-1}
$$

By Jensen's inequality $c_{2} / \bar{c}^{2} \geq 1$ with equality if $\boldsymbol{\Theta}=\mathbf{I}_{M}$. The computation of (18) involves the evaluation of only one fixed-point equation, given by (22).

Corollary 1: Let $\boldsymbol{\Theta}=\mathbf{I}_{M}$ and $\mathbf{L}=\mathbf{I}_{K}$ then $\gamma_{k, \mathrm{zf}}^{\circ}$ takes the explicit form

$$
\gamma_{\mathrm{zf}}^{\circ} \triangleq \gamma_{k, \mathrm{zf}}^{\circ}=\frac{1-\tau^{2}}{\tau^{2}+\frac{1}{\rho}}(\beta-1)
$$

Proof of Corollary 1: By substituting $\Theta=\mathbf{I}_{M}$ and $\mathbf{L}=$ $\mathbf{I}_{K}$ into (22), $\bar{c}$ is explicitly given by $\bar{c}=(\beta-1) / \beta$. Since $c_{2} / \bar{c}^{2}=1$ we have $\bar{\Psi}^{\circ}=\bar{\Upsilon}^{\circ}=1 /(\beta-1)$.

Proof of Theorem 2: The SINR $\gamma_{k, \mathrm{rzf}}$ of user $k$ of RZF for large $(K, M)$ is given by [6]

$$
\gamma_{k, \mathrm{rzf}}=\frac{l_{k}^{2}\left(1-\tau^{2}\right) m_{\mathbf{A}}^{2}}{l_{k} \Upsilon\left(1-\tau^{2}\left[1-\left(1+l_{k} m_{\mathbf{A}}\right)^{2}\right]\right)+\frac{\Psi(\alpha)}{\rho}\left(1+l_{k} m_{\mathbf{A}}\right)^{2}}
$$

where $\mathbf{A}=\hat{\mathbf{X}}^{\mathrm{H}} \mathbf{L} \hat{\mathbf{X}}+\alpha \boldsymbol{\Theta}^{-1}$ and

$$
\begin{aligned}
m_{\mathbf{A}} & \triangleq m_{\mathbf{A}}(0)=\operatorname{Tr} \mathbf{A}^{-1} \\
\Upsilon & =m_{\mathbf{A}}-\alpha \operatorname{Tr} \boldsymbol{\Theta}^{-1} \mathbf{A}^{-2} \\
\Psi(\alpha) & =\operatorname{Tr} \hat{\mathbf{H}}^{\mathrm{H}} \hat{\mathbf{H}}\left(\hat{\mathbf{H}}^{\mathrm{H}} \hat{\mathbf{H}}+\alpha \mathbf{I}_{M}\right)^{-2}
\end{aligned}
$$

The underlying strategy is as follows: The terms in the SINR of RZF that depend on $\alpha$, i.e. $m_{\mathbf{A}}, \Upsilon$ and $\Psi$, are expanded around $\alpha=0$. Subsequently we take the limit $\gamma_{k, \mathrm{zf}}=$ $\lim _{\alpha \rightarrow 0} \gamma_{k, \text { rzf }}$. Finally, we find the deterministic equivalent $\gamma_{k, \mathrm{zf}}^{\circ}$.

We expand $m_{\mathbf{A}}=\operatorname{Tr} \mathbf{A}^{-1}$ around $\alpha=0$ as follows

$$
\begin{aligned}
& m_{\mathbf{A}} \stackrel{(a)}{=} \frac{1}{\alpha} \operatorname{Tr} \boldsymbol{\Theta}-\frac{1}{\alpha M} \operatorname{tr} \hat{\mathbf{H}} \Theta \hat{\mathbf{H}}^{\mathrm{H}}\left(\hat{\mathbf{H}} \hat{\mathbf{H}}^{\mathrm{H}}+\alpha \mathbf{I}_{K}\right)^{-1} \\
& \stackrel{(b)}{\approx} \frac{1}{\alpha} \operatorname{Tr} \boldsymbol{\Theta}-\frac{1}{\alpha M} \operatorname{tr} \hat{\mathbf{H}} \Theta \hat{\mathbf{H}}^{\mathrm{H}}\left[\left(\hat{\mathbf{H}} \hat{\mathbf{H}}^{\mathrm{H}}\right)^{-1}-\alpha\left(\hat{\mathbf{H}} \hat{\mathbf{H}}^{\mathrm{H}}\right)^{-2}\right]
\end{aligned}
$$

where $(a)$ follows from the matrix inversion lemma (MIL) and in $(b)$ we rewrite the inverse in terms of a Taylor series of order 2 around the point $\alpha=0$. In step $(b)$ it is necessary to assume that $\beta>1$ to assure that the maximum eigenvalue of matrix $\left(\hat{\mathbf{H}} \hat{\mathbf{H}}^{\mathrm{H}}\right)^{-1}$ is bounded for all large $M$. For $\alpha \operatorname{Tr} \boldsymbol{\Theta}^{-1} \mathbf{A}^{-2}$ we obtain

$$
\begin{aligned}
\alpha \operatorname{Tr} \Theta^{-1} \mathbf{A}^{-2} & \approx \frac{1}{\alpha} \operatorname{Tr} \Theta-\frac{1}{\alpha M} \operatorname{tr} \hat{\mathbf{H}} \Theta \hat{\mathbf{H}}^{\mathrm{H}}\left(\hat{\mathbf{H}} \hat{\mathbf{H}}^{\mathrm{H}}\right)^{-1} \\
& +\frac{\alpha}{M} \operatorname{tr} \hat{\mathbf{H}} \boldsymbol{\Theta} \hat{\mathbf{H}}^{\mathrm{H}}\left(\hat{\mathbf{H}} \hat{\mathbf{H}}^{\mathrm{H}}\right)^{-3} .
\end{aligned}
$$

Substituting (29) and (30) into (26) and taking the limit $\alpha \rightarrow 0$, we obtain

$$
\bar{\Upsilon}=\lim _{\alpha \rightarrow 0} \Upsilon=\frac{1}{M} \operatorname{tr} \hat{\mathbf{H}} \Theta \hat{\mathbf{H}}^{\mathrm{H}}\left(\hat{\mathbf{H}} \hat{\mathbf{H}}^{\mathrm{H}}\right)^{-2} .
$$

Replacing $m_{\mathbf{A}}, \Upsilon$ and $\Psi(\alpha)$ in (24) with (29), (31) and $\bar{\Psi}=$ $\Psi(0)$, respectively, we have

$$
\gamma_{k, \mathrm{zf}}=\lim _{\alpha \rightarrow 0} \gamma_{k, \mathrm{rzf}}=\frac{1-\tau^{2}}{l_{k} \tau^{2} \bar{\Upsilon}+\frac{\bar{\Psi}}{\rho}} .
$$

Now we derive a deterministic equivalent $\bar{\Psi}^{\circ}$ and $\bar{\Upsilon}^{\circ}$ for $\bar{\Psi}$ and $\bar{\Upsilon}$, respectively.

Applying Theorem 1 we find $\bar{\Psi}^{\circ}$ s.t. $\bar{\Psi}-\bar{\Psi}^{\circ} \stackrel{M \rightarrow \infty}{\longrightarrow} 0$ almost surely, as

$$
\bar{\Psi}^{\circ}=m_{\hat{\mathbf{H}} \hat{\mathbf{H}}^{H}}^{\circ}(0)=\frac{1}{\beta \bar{c}} \operatorname{Tr} \mathbf{L}^{-1},
$$

where $\bar{c}$ is defined in (22).

In order to find $\bar{\Upsilon}^{\circ}$ notice that, we can diagonalize $\Theta$ in (31) s.t. $\boldsymbol{\Theta}=\mathbf{U} \operatorname{diag}\left(\lambda_{1}, \ldots, \lambda_{M}\right) \mathbf{U}^{\mathrm{H}}$ and still have i.i.d. elements in the $k$ th column $\hat{\mathbf{x}}_{k}^{\prime}$ of $\hat{\mathbf{X}} \mathbf{U}$. Denoting $\mathbf{C}=\hat{\mathbf{H}} \hat{\mathbf{H}}^{\mathrm{H}}$ and $\mathbf{C}_{[k]}=$ $\hat{\mathbf{H}}_{[k]} \hat{\mathbf{H}}_{[k]}^{\mathrm{H}}-\lambda_{k} \mathbf{L}^{1 / 2} \hat{\mathbf{x}}_{k}^{\prime} \hat{\mathbf{x}}_{k}^{\prime H} \mathbf{L}^{1 / 2}$ and applying [14, Lemma 2.2] twice, equation (31) takes the form

$$
\bar{\Upsilon}=\frac{1}{M} \sum_{k=1}^{M} \lambda_{k}^{2} \frac{\hat{\mathbf{x}}_{k}^{\prime H} \mathbf{L}^{1 / 2} \mathbf{C}_{[k]}^{-2} \mathbf{L}^{1 / 2} \hat{\mathbf{x}}_{k}^{\prime}}{\left(1+\lambda_{k} \hat{\mathbf{x}}_{k}^{\prime H} \mathbf{L}^{1 / 2} \mathbf{C}_{[k]} \mathbf{L}^{1 / 2} \hat{\mathbf{x}}_{k}^{\prime}\right)^{2}} .
$$

Applying [14, Lemma 3.1] together with $\operatorname{Tr} \mathbf{C}_{[k]}^{-1} \quad-$ $\operatorname{Tr}^{-1} \stackrel{M \rightarrow \infty}{\longrightarrow} 0$ [6], we obtain

$$
\bar{\Upsilon}-\frac{1}{\beta} \operatorname{Tr} \mathbf{L} \mathbf{C}^{-2} \frac{1}{M} \sum_{k=1}^{M} \frac{\lambda_{k}^{2}}{\left(1+\lambda_{k} \frac{1}{\beta} \operatorname{Tr} \mathbf{L} \mathbf{C}^{-1}\right)^{2}} \stackrel{M \rightarrow \infty}{\longrightarrow} 0
$$

almost surely. To determine a deterministic equivalent $m_{\mathbf{C , \mathbf { L }}}(0)^{\circ}$ for $m_{\mathbf{C , \mathbf { L }}}(0)=\operatorname{Tr} \mathbf{L} \mathbf{C}^{-1}$, we apply Theorem 1 as for (33). For $\operatorname{Tr} \mathbf{L} \mathbf{C}^{-2}$ we have

$$
\operatorname{Tr} \mathbf{L} \mathbf{C}^{-2}=m_{\mathbf{C}^{2}, \mathbf{L}}(z)=\left.\frac{\partial m_{\mathbf{C}^{2}, \mathbf{L}}(z)}{\partial z}\right|_{z=0}=m_{\mathbf{C}, \mathbf{L}}^{\prime}(0) .
$$

The derivative of $m_{\mathbf{C , L}}(0)^{\circ}$ is a deterministic equivalent of $m_{\mathbf{C}, \mathbf{L}}^{\prime}(0)$, so that applied to (35), we have a deterministic equivalent $\bar{\Upsilon}^{\circ}$, s.t. $\bar{\Upsilon}-\bar{\Upsilon}^{\circ} \stackrel{M \rightarrow \infty}{\longrightarrow} 0$ almost surely, that verifies

$$
\bar{\Upsilon}^{\circ}=\frac{c_{2} / \bar{c}^{2}}{\beta-c_{2} / \bar{c}^{2}} \operatorname{Tr} \mathbf{L}^{-1}
$$

where $\bar{c}$ and $c_{2}$ are defined in (22) and (21), respectively. Finally, we obtain (18) by substituting $\bar{\Psi}$ and $\bar{\Upsilon}$ in (32) by their respective deterministic equivalents (33) and (37), which completes the proof.

\section{Asymptotically Optimal Number Of Users $K$}

In general, for fixed $\boldsymbol{\Theta}, \mathbf{L}, \rho$ and $\tau^{2}$, consider the problem of finding the optimal number of users $K^{\star \circ}$ (or equally $\left.\beta^{\star \circ}=M / K^{\star \circ}\right)$, such that the approximated sum rate $R_{\text {sum }}^{\circ} \triangleq$ $\sum_{k=1}^{K} \log \left(1+\gamma_{k, \mathrm{zf}}^{\circ}\right)$ is maximized, i.e.

$$
\beta^{\star \circ}=\underset{\beta>1}{\arg \max } \frac{1}{\beta} \int \log \left(1+\gamma_{k, \mathrm{zf}}^{\circ}\right) d F^{\mathbf{L}}(l),
$$

where we suppose that the user channel gains $l_{k}$ are distributed according to some probability distribution function $F^{\mathbf{L}}$. By 
setting the derivative of (38) w.r.t. $\beta$ to zero, we obtain the implicit equation

$$
\beta \int \frac{\frac{\partial \gamma_{k, \mathrm{zf}}^{\circ}}{\partial \beta} d F^{\mathbf{L}}(l)}{1+\gamma_{k, \mathrm{zf}}^{\circ}}=\int \log \left(1+\gamma_{k, \mathrm{zf}}^{\circ}\right) d F^{\mathbf{L}}(l) .
$$

Thus, $\beta^{\star \circ}$ is the solution to (39).

In the special case of $\boldsymbol{\Theta}=\mathbf{I}_{M}$ and $\mathbf{L}=\mathbf{I}_{K}$, the SINR $\gamma_{k, \mathrm{zf}}^{\circ}$ is given in Corollary 1 and the solution to (39) has an explicit form. For equation (39) we obtain

$$
\frac{a \beta}{1+a(\beta-1)}=\log (1+a(\beta-1))
$$

where $a=\frac{1-\tau^{2}}{\tau^{2}+\frac{1}{\rho}}$. Denoting

$$
w(\beta)=\frac{a-1}{a(\beta-1)+1} \quad \text { and } \quad x=\frac{a-1}{e},
$$

we can rewrite (40) as

$$
w(\beta) e^{w(\beta)}=x .
$$

Notice that $w(\beta)=\mathcal{W}(x)$, where $\mathcal{W}(x)$ is the Lambert Wfunction defined as $z=\mathcal{W}(z) e^{\mathcal{W}(z)}, z \in \mathbb{C}$. Therefore, by solving $w(\beta)=\mathcal{W}(x)$ we have

$$
\beta^{\star \circ}=\left(1-\frac{1}{a}\right)\left(1+\frac{1}{\mathcal{W}(x)}\right) .
$$

For $\tau \in[0,1], \beta>1$ we have $w \geq-1$ and $x \in\left[-e^{-1}, \infty\right)$. In this case $\mathcal{W}(x)$ is a single-valued function. If $\tau=0$, we obtain the results in [5]. Note that only rational values of $\beta$ are meaningful in practice.

If the transmit antennas are spaced sufficiently apart the major loss in sum rate is due to path loss, cf. Figure 1. Therefore, it is of interest to characterize the sum rate gap $R_{\Delta}$ between a user distribution $F^{\mathbf{L}}$ and equally distant users $\mathbf{L}=\mathbf{I}_{K}$. For a fixed $\beta$ and with $\tau^{2}=0$, we have

$$
R_{\Delta}=K \log \left(\frac{1+\rho(\beta-1)}{1+\frac{\rho}{\Psi^{\circ}}}\right) .
$$

Although $\bar{\Psi}^{\circ}$ induces a significant loss in sum rate, we still have a linear scaling of the sum rate with SNR [dB] because $\bar{\Psi}^{\circ}$ is independent of the SNR. Since $\Theta$ has only a minor impact on $R_{\Delta}$, for reasonable antenna separations, we obtain for $\boldsymbol{\Theta}=\mathbf{I}_{M}$ and asymptotically high SNR

$$
R_{\Delta}^{\lim }=\lim _{\rho \rightarrow \infty} R_{\Delta}=K \log \operatorname{Tr} \mathbf{L}^{-1} .
$$

From (45) we notice that $R_{\Delta}^{\lim }$ is solely depending on the distribution of the channel gains $\mathbf{L}$. As an example we suppose that the $K$ users are uniformly distributed on a ring of maximal and minimal radius $r_{\max }$ and $r_{\min }$, respectively. Furthermore, denoting $d_{k}$ the distance from user $k$ to the transmitter, we apply the exponential path loss model (indicated by the notation $\left.\mathbf{L} \neq \mathbf{I}_{K}\right)$ i.e. $l_{k}=\kappa d_{k}^{-\alpha}$ where $\kappa$ is chosen s.t. $E l_{k}=1$ for given $r_{\max }, r_{\min }$ and $\alpha$. This normalization ensures a fair

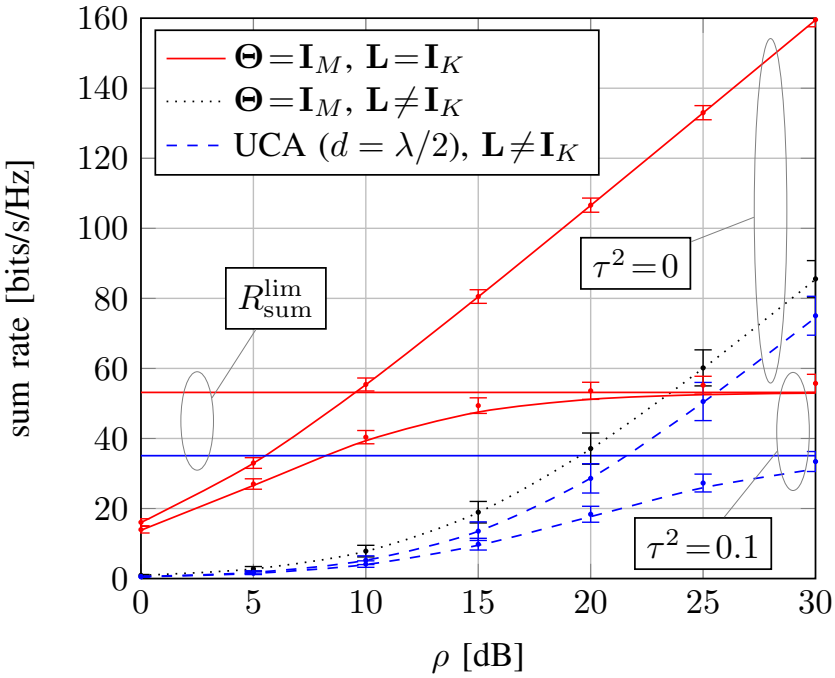

Fig. 1. ZF, sum rate vs. SNR with $M=32, \beta=2$ simulation results are indicated by circle marks with error bars indicating one standard deviation in each direction.

comparison to the scenario $\mathbf{L}=\mathbf{I}_{K}$. Assuming $r_{\max } \gg r_{\min }$, we obtain

$$
R_{\Delta}^{\lim } \approx K \log \left(\frac{4}{\alpha^{2}-4}\right)+(\alpha-2) K \log \left(\frac{r_{\max }}{r_{\min }}\right)
$$

Therefore, the sum rate gap $R_{\Delta}^{\lim }$ increases with $\alpha$ and $\log r_{\max }$ for a fixed $r_{\min }$.

\section{NumericAl RESUlts}

In our simulations all results are averaged over 10,000 independent Rayleigh block-fading channel realizations.

The transmit correlation is assumed to depend only on the distance $d_{i j}, i, j=1,2, \ldots, M$ between antennas $i$ and $j$ placed on a uniform circular array (UCA). Thus, $(\boldsymbol{\Theta})_{i j}=$ $\mathrm{J}_{0}\left(2 \pi d_{i j} / \lambda\right)$ [15], where $\mathrm{J}_{0}$ is the zero-order Bessel function of the first kind and $\lambda$ is the signal wavelength. To ensure that $\lambda_{M}$ grows slower than $O(M)$, we suppose that the distance between adjacent antennas $d=d_{i, i+1}$ is independent of $M$, i.e. as $M$ grows the radius of the UCA increases.

Furthermore, we consider that the users are distributed uniformly on a ring with $r_{\max }=500 \mathrm{~m}$ and $r_{\max }=35 \mathrm{~m}$ with $\alpha=3.5$, [16] ("Suburban Macro") and $\kappa$ s.t. $\operatorname{Tr} \mathbf{L}=1$.

Figure 1 compares the sum rate performance of the approximated sum rate to Monte-Carlo simulations. We observe, that the expressions derived for large $(K, M)$ lie approximately within the band of two standard deviations of the simulation results even for finite $(K, M)$. Therefore, the approximation derived in Theorem 2 are accurate and can be applied to concrete optimization problems for the multi-user downlink channel. For high SNR, the sum rate loss due to path loss is given by (46), $R_{\Delta}^{\lim } \approx 75[\mathrm{bits} / \mathrm{s} / \mathrm{Hz}]$, corresponding well to the simulation results.

Figure 2 compares the optimal number of users $K^{\star 0}$ in (43) to the optimal number of users $K^{\star}$ obtained from MonteCarlo simulations. More precisely $K^{\star}$ is the number of users 


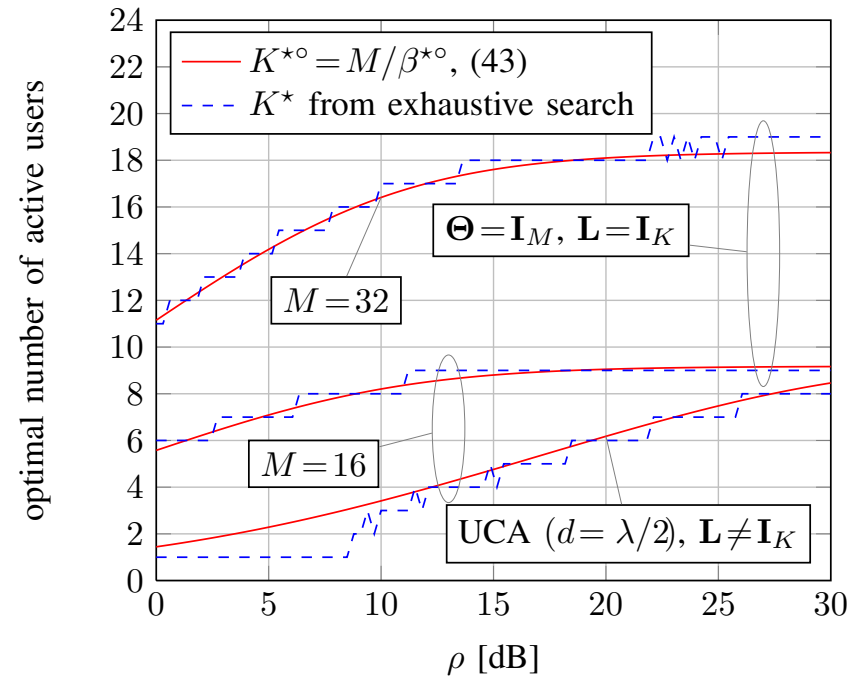

Fig. 2. ZF, sum rate maximizing number of active users vs. SNR with $\tau^{2}=0.1$

$K<M$ that maximizes the ergodic sum rate, when distributed uniformly over the ring defined above. It can be observed that $K^{\star \circ}$ predicted by the asymptotic results does fit well even for finite dimensions. Moreover, introducing correlation and path loss leads to larger dispersion of the optimal $K$ over the selected SNR range.

Figure 3 depicts the impact of the number of served users on the ergodic sum rate. It can be observed that $K^{\star \circ}$ achieves most of the sum rate even for finite $(K, M)$ and thus, is a good choice for the user allocation at the transmitter. Moreover, we observe that adapting the number of users is beneficial compared to a fixed $K$. From Figure 2 we identify $K=8$ as a good choice (for $\tau^{2}=0.1$ ) and, as expected, the performance is optimal in the medium SNR regime and suboptimal at low and high SNR. The situation changes by adding correlation and path loss. Since $K=8$ is highly suboptimal for low and medium SNR (cf. Figure 2) we observe a significant loss in sum rate in this regime.

\section{CONCLUSION}

In this paper we derived deterministic equivalent of the SINR of ZF precoding by applying recent results from random matrix theory. These approximations are shown to be very accurate even for finite dimensions and thus provide useful tools for many engineering applications. In particular the approximated sum rate enabled us to derive expressions for the optimal number of users in the cell and to characterize the impact of a spatial user density on the achievable sum rate.

\section{REFERENCES}

[1] H. Weingarten, Y. Steinberg, and S. Shamai, "The Capacity Region of the Gaussian Multiple-Input Multiple-Output Broadcast Channel," IEEE Trans. Inf. Theory, vol. 52, no. 9, pp. 3936-3964, Sep. 2006.

[2] G. Caire and S. Shamai, "On the Achievable Throughput of a Multiantenna Gaussian Broadcast Channel," IEEE Trans. Inf. Theory, vol. 49, no. 7, pp. 1691-1706, Jul. 2003.

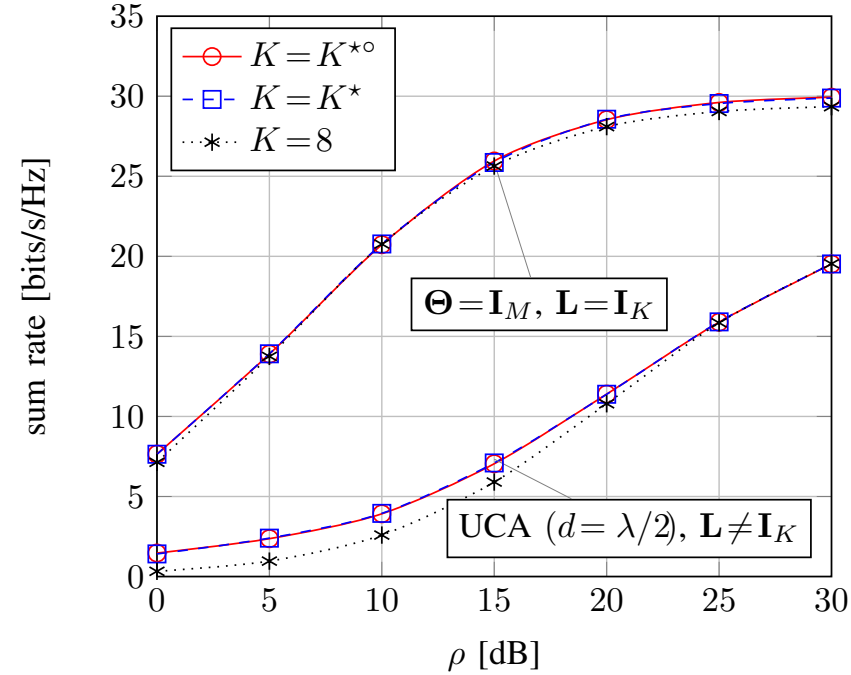

Fig. 3. ZF, ergodic sum rate vs. SNR with $M=16$ and $\tau^{2}=0.1$.

[3] C. B. Peel, B. M. Hochwald, and A. L. Swindlehurst, "A VectorPerturbation Technique for Near-Capacity Multiantenna Multiuser Communication-Part I: Channel Inversion and Regularization," IEEE Trans. Commun., vol. 53, no. 1, pp. 195-202, Jan. 2005.

[4] H. Viswanathan and S. Venkatesan, "Asymptotics of Sum Rate for Dirty Paper Coding and Beamforming in Multiple-Antenna Broadcast Channels," in Proc. IEEE Annual Allerton Conference on Communication, Control, and Computing, Monticello, Illinois, Oct. 2003, pp. 1064-1073.

[5] B. Hochwald and S. Vishwanath, "Space-Time Multiple Access: Linear Growth in the Sum Rate," in Proc. IEEE Annual Allerton Conference on Communication, Control, and Computing, Monticello, Illinois, Oct. 2002, pp. 387-396.

[6] S. Wagner, R. Couillet, M. Debbah, and D. T. M. Slock, "Large System Analysis of Linear Precoding in MISO Broadcast Channels with Limited Feedback," IEEE Trans. Inf. Theory, submitted for publication. [Online]. Available: http://arxiv.org/abs/0906.3682

[7] A. M. Tulino and S. Verdú, Random Matrix Theory and Wireless Communications. Delft, Netherlands: Now Publishers Inc., 2004.

[8] C.-N. Chuah, D. N. C. Tse, J. M. Kahn, and R. A. Valenzuela, "Capacity Scaling in MIMO Wireless Systems Under Correlated Fading," IEEE Trans. Inf. Theory, vol. 48, no. 3, pp. 637-650, Mar. 2002.

[9] A. M. Tulino, A. Lozano, and S. Verdú, "Impact of Antenna Correlation on the Capacity of Multiantenna Channels," IEEE Trans. Inf. Theory, vol. 51, no. 7, pp. 2491-2509, Jul. 2005.

[10] H. Shin, M. Z. Win, and M. Chiani, "Asymptotic Statistics of Mutual Information for Doubly Correlated MIMO Channels," IEEE Trans. Wireless Commun., vol. 7, no. 2, pp. 562-573, Feb. 2008.

[11] A. D. Dabbagh and D. J. Love, "Multiple Antenna MMSE Based Downlink Precoding with Quantized Feedback or Channel Mismatch," IEEE Trans. Commun., vol. 56, no. 11, pp. 1859-1868, Nov. 2008.

[12] T. Yoo and A. Goldsmith, "Capacity and Power Allocation for Fading MIMO Channels with Channel Estimation Error," IEEE Trans. Inf. Theory, vol. 52, no. 5, pp. 2203-2214, May 2006.

[13] A. A. Hutter, E. de Carvalho, and J. M. Cioffi, "On the Impact of Channel Estimation for Multiple Antenna Diversityreception in Mobile OFDM Systems," in Proc. IEEE Conference Record of the Asilomar Conference on Signals, Systems, and Computers, vol. 2, Pacific Grove, CA, Nov. 2000, pp. 1820-1824.

[14] J. W. Silverstein and Z. D. Bai, "On the Empirical Distribution of Eigenvalues of a Class of Large Dimensional Random Matrices," Journal of Multivariate Analysis, vol. 54, no. 2, pp. 175-192, Aug. 1995.

[15] W. C. Jakes and D. C. Cox, Microwave Mobile Communications. Hoboken, NJ: Wiley-IEEE Press, 1994.

[16] H. Huang, "TR 25.996 Spatial Channel Model for Multiple Input Multiple Output (MIMO) Simulations," 3GPP, Tech. Rep., Dec. 2008. 